\title{
Tumor maligno de la vaina nerviosa periférica del nervio vago: Reporte de un caso
}

\author{
Malignant tumor of the peripheral nerve sheath of the vagus nerve: \\ A case report
}

Felipe Parentini H. ${ }^{1}$, José Rojas B. ${ }^{1}$, Francisca Fernández A. ${ }^{2,3}$, Jaime Bermeo S. ${ }^{2}$

'Servicio de Otorrinolaringología, Hospital Carlos Van Buren, Universidad de Valparaíso. Valparaíso, Chile. ${ }^{2}$ Servicio de Otorrinolaringología, Hospital Carlos Van Buren. Valparaíso, Chile. ${ }^{3}$ Departamento de Especialidades, Cátedra de Otorrinolaringología, Escuela de Medicina, Facultad de Medicina, Universidad de Valparaíso. Viña del Mar, Chile.

Los autores declaran no tener conflictos de interés.

Recibido el 8 de julio de 2020 Aceptado el 7 de octubre de 2020

Correspondencia: Francisca Fernández A.

Cuatro $1 / 2$ poniente 472 Viña del Mar, Chile. Email: francisca.fernandez@uv.cl

\section{Resumen}

Los tumores malignos de la vaina nerviosa forman parte del 5\% de los sarcomas de partes blandas. Si bien son infrecuentes, su incidencia aumenta en caso de neurofibromatosis tipo 1. Cuando estos tumores están ubicados en cabeza y cuello, suelen ser asintomáticos, por lo que su diagnóstico es tardío. El tratamiento es principalmente quirúrgico, con una tasa de recidiva importante y pobre sobrevida a los 5 años. Se presenta el reporte de un caso de una paciente de 52 años con antecedentes de neurofibromatosis tipo 1 y un tumor maligno de vaina nerviosa periférica del nervio vago, localizado en el espacio parafaríngeo.

Palabras clave: Tumor maligno de la vaina del nervio periférico, neurofibromatosis tipo 1, espacio parafaríngeo, nervio vago.

\begin{abstract}
Malignant peripheral nerve sheath tumors are part of 5\% of soft tissue sarcomas. Although they are infrequent, their incidence increases in case of neurofibromatosis type 1. When these tumors are located in the head and neck, they are usually asymptomatic, so their diagnosis is delayed. Treatment is primarily surgical, with a significant recurrence rate and poor 5-year survival. We present a case report of a 52-year-old patient with a history of type 1 neurofibromatosis and a malignant peripheral nerve sheath tumor of the vagus, located in the parapharyngeal space.

Keywords: Malignant peripheral nerve sheath tumors, neurofibromatosis type 1, parapharyngeal space, vagus nerve.
\end{abstract}

\section{Introducción}

Los tumores malignos de la vaina del nervio periférico (TMVNP), fueron descritos por primera vez en 1935 como neurilemoma maligno. También llamado schwannoma maligno, neurofibrosarcoma y sarcoma neurogénico. El término "tumores malignos de la vaina del nervio periférico" fue introducido por la OMS para reemplazar todos los términos previos que pudiesen ser confundentes ${ }^{1,2}$. Refiriéndose a las neoplasias de los tejidos blandos que surgen de los nervios periféricos y muestran una diferenciación variable hacia uno de los componentes celulares de la vaina del nervio periférico.

Los TMVNP son responsables del 5\% de los sarcomas de partes blandas y su incidencia es de $0,001 \%$, la cual aumenta a un $10 \%$ en caso de neurofibromatosis tipo $1(\mathrm{NF} 1)^{1-3}$. La incidencia es similar en ambos sexos y la edad de presentación fluctúa entre los 20 y los 50 años, observándose aproximadamente 10 años antes en los pacientes con NF1. Suelen generarse de novo, sin embargo, un 10\% tiene antecedentes de exposición a radiación, con una latencia de aparición de 10 a 40 años. Además, se ha descrito asociación entre $\mathrm{NF}^{1}$ y transformación maligna de neurofibromas o schwannomas ${ }^{2,4-6}$. 


\section{Caso Clínico}

Mujer de 52 años con antecedentes de cáncer de mama izquierda en tratamiento y neurofibromatosis tipo 1. Consulta por cuadro de 2 años de evolución de molestias cervicales inespecíficas, que en los últimos 6 meses se agrega disfonía y disfagia progresiva, con baja de peso de 22 kilos. Al examen físico destaca múltiples manchas café con leche de $1 \mathrm{a} 2 \mathrm{~cm}$ en tronco y extremidades, asociado a neurofibromas en tronco y cara. $\mathrm{Al}$ examen otorrinolaringológico, se observa aumento de volumen del paladar blando y pared lateral orofaríngea a derecha. En la nasofibroscopía, destaca un aumento de volumen submucoso en pared lateral derecha de rinofaringe, de bordes lisos, no pulsátil, que traspasa línea media, sin lograr visualizar en profundidad dado el efecto de masa e intolerancia de la paciente. Se realiza estudio complementario con resonancia magnética, donde se visualiza un proceso expansivo sólido de 5,2 × 4,5 × 3,5 $\mathrm{cm}$, ubicado en el espacio parafaríngeo derecho, de límites bien definidos, hiperintenso en $\mathrm{T} 1$ y T2, con componentes que refuerzan con gadolinio. El efecto de masa provocado por el tumor, determina una estenosis de la vía aérea, y desplazamiento de la carótida interna hacia medial y carótida externa hacia lateral y posterior. En la angiografía de cuello y cerebro por tomografía computada (angio-TC), se logra apreciar de mejor forma la interposición entre las carótidas interna y externa en el plano axial (Figura 1), descartando un origen glómico por su falta de irrigación.

Con estos antecedentes, se decide realizar una resección quirúrgica mediante un abordaje transcervicofacial con mandibulotomía paramedial en escalón, con el objetivo de lograr un adecuado control de los grandes vasos durante la resección. Realizándose la enucleación completa del tumor y cierre de la mandibulotomía con dos placas de titanio 2.0 y cuatro tornillos bicoronales. La biopsia informa una masa nodular sólida de 7,5 x 5,0 x $5,0 \mathrm{~cm}$, limitado por fina cápsula fibrosa, con algunas pequeñas áreas de necrosis, compatibles con un tumor maligno de vaina de nervio periférico (Figura 2), correspondiente a un tumor grado 2 de acuerdo con el sistema de clasificación propuesto por el French Federation of Cancer Centers Sarcoma Group (FFCCSG).
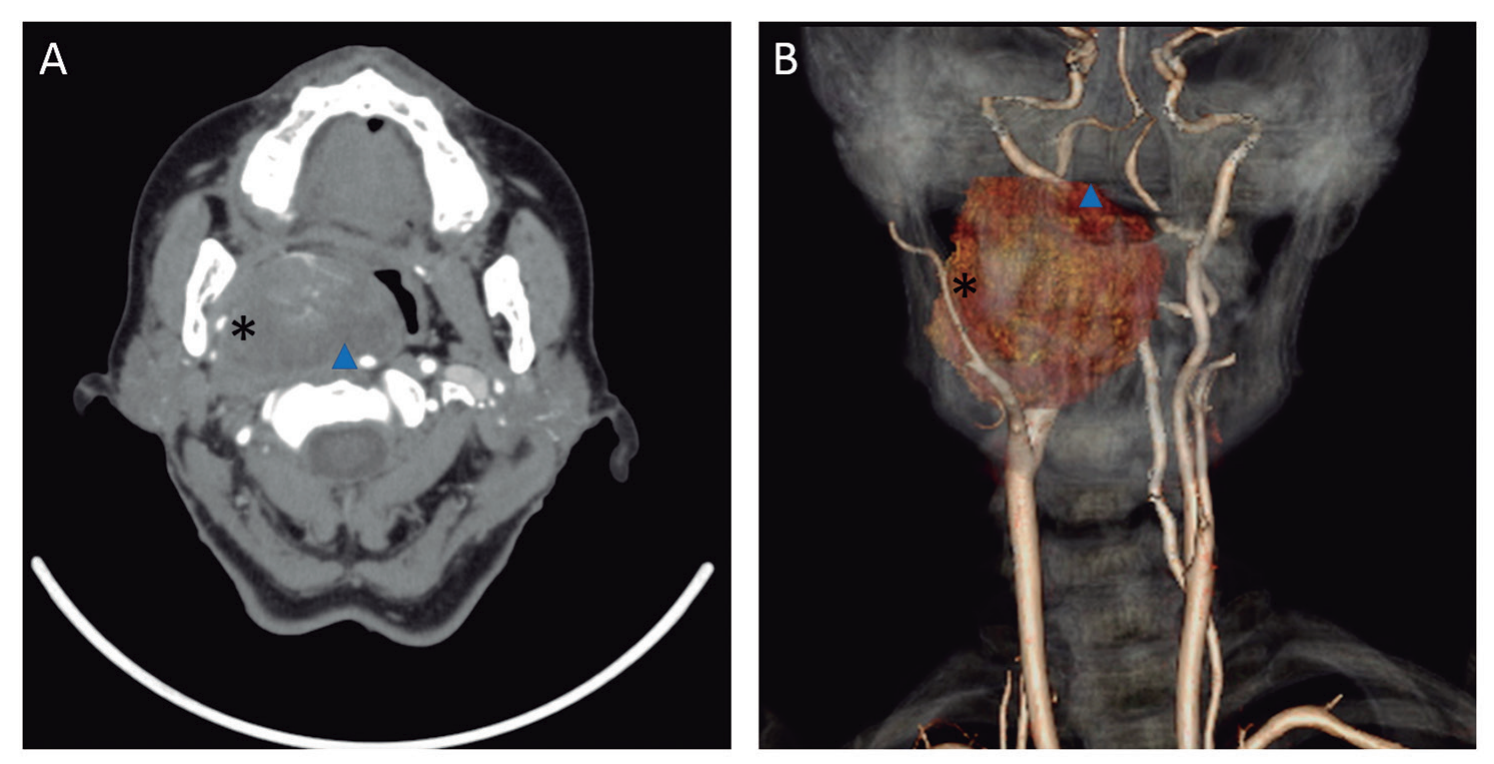

Figura 1. Lesión tumoral en bifurcación carotídea, desplazando carótida interna hacia medial y carótida externa hacia lateral y posterior. Angiografía de cuello por tomografía computada en su plano axial (A) y reconstrucción 3D (B). Asterisco indica carótida externa, triángulo indica carótida interna. 


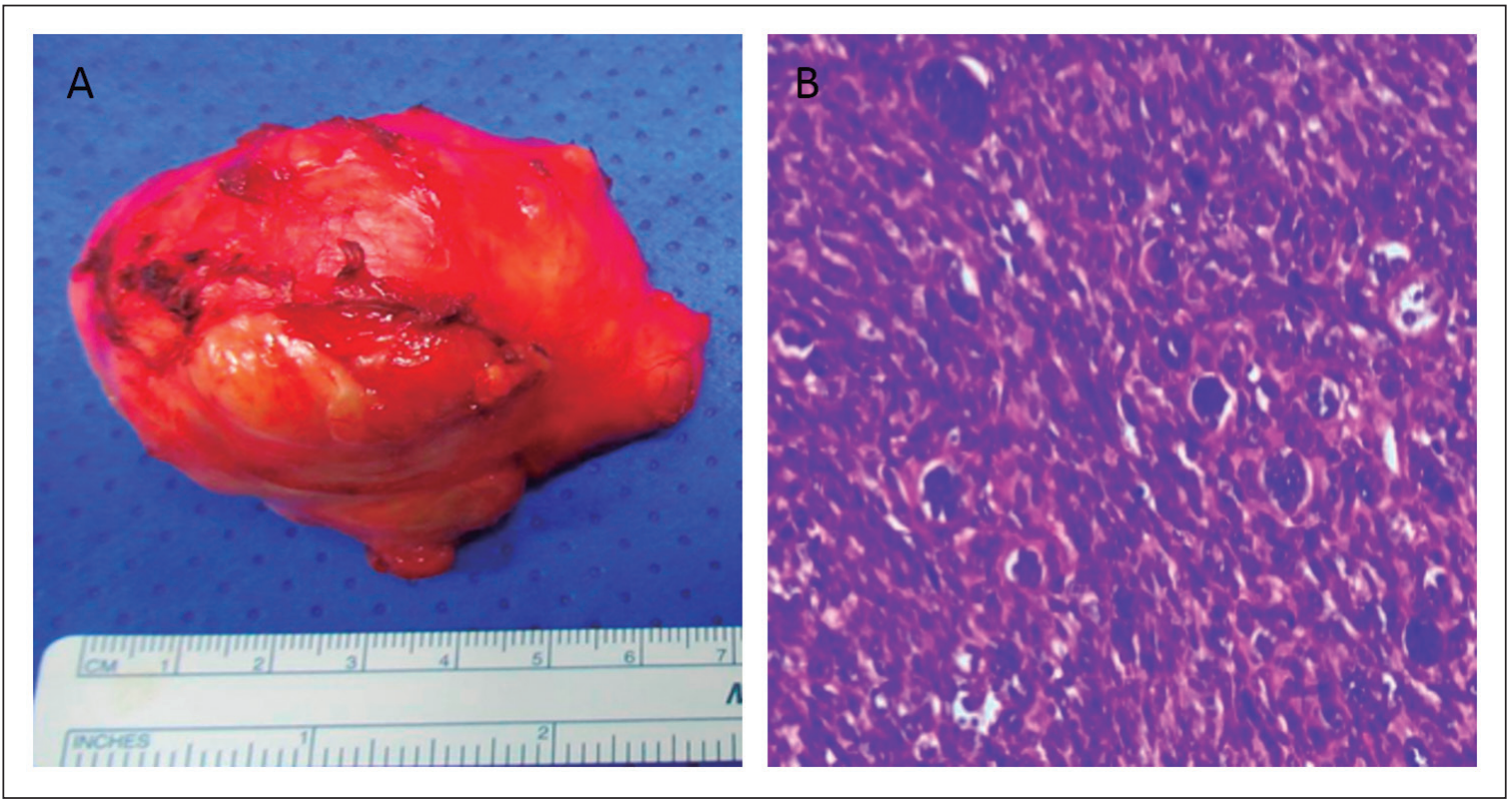

Figura 2. Tumor maligno de vaina de nervio periférico. A: Imagen macroscópica de la masa resecada y B: sección de la tinción de hematoxilina-eosina de la muestra obtenida en la biopsia de la masa.

\section{Discusión}

El 20\% de los TMVNP se ubican en cabeza y cuello y suelen presentarse como una masa de crecimiento rápido asociada a dolor, parestesias y déficit neurológico ${ }^{7}$. En caso de déficit, los hallazgos pueden ser disfonía, disnea, disfagia, tos, sensación de cuerpo extraño faríngeo y síndrome de Horner ${ }^{8}$. Para complementar el estudio, el apoyo imagenológico será fundamental, siendo la resonancia magnética el examen de elección para detectar TMVNP, con una sensibilidad del 67\% y especificidad del $97 \%{ }^{9}$. Clásicamente se pueden observar márgenes irregulares, edema perilesional, degeneración quística, realce heterogéneo en T1 y captación irregular de contraste en $\mathrm{T} 1^{7-10}$. En nuestro paciente, las imágenes eran sugerentes de un tumor benigno, por los márgenes bien definidos y captación homogénea en T1. La angio-TC nos permitirá evaluar la irrigación y relación con los grandes vasos. Un tumor avascular sugerirá un schwannoma. La localización de los TMVNP en la bifurcación carotídea, nos hará sospechar un origen en la cadena simpático cervical y la separación de la vena yugular interna y arteria carótida interna sin ensanchamiento de la bifurcación carotídea, un origen en el nervio vago ${ }^{8,11}$. En este caso, el ensanchamiento de la bifurcación carotídea debe hacernos sospechar un origen glómico. Sin embargo, su característica avascular y síntomas asociados nos inclinó hacia un schwannoma. Por otro lado, la citología por punción con aguja fina ha ganado gran relevancia en el estudio prequirúrgico del TMVNP, donde se observan células fusiformes, de contornos irregulares, con ligera irregularidad nuclear y ocasionales mitosis ${ }^{1}$. En este caso se decidió no puncionar por su cercanía a los grandes vasos. Al no existir criterios morfológicos, inmunohistoquímicos y moleculares específicos, el diagnóstico histológico será difícil. La correlación con la historia clínica, incluyendo el antecedente de neurofibromatosis tipo 1 es fundamental. Macroscópicamente, los tumores pueden estar dentro o adheridos a un tronco nervioso, neurofibroma o neurofibroma plexiforme. A menudo tienen una apariencia fusiforme y pueden extenderse dentro de un nervio. Las lesiones tienden a ser blancas y sólidas, a veces con cambios mixoides, con frecuentes necrosis y hemorragias. Microscópicamente, suelen ser lesiones altamente 
infiltrantes que muestran una variada gama de morfologías celulares (que incluyen huso, epitelioide, pleomórfico o células redondas pequeñas) y patrones arquitectónicos ${ }^{6}$. Dentro de los factores de mal pronóstico se encuentran: localización en cabeza y cuello, ubicación profunda, tamaño $>5 \mathrm{~cm}$, irradiación previa, márgenes quirúrgicos positivos y la asociación con $\mathrm{NF}^{6-7}$. Lamentablemente nuestra paciente presenta múltiples factores de mal pronóstico.

Un $40 \%$ de los pacientes con TMVNP tendrán recurrencias locales, aumentando el riesgo de desarrollar nuevas recurrencias y metástasis a distancia, siendo la localización más frecuente a nivel pulmonar ${ }^{2,5}$. La presencia de recurrencia local incrementa la mortalidad 2,5 veces. La sobrevida global a 5 años disminuye de $30 \%-40 \%$ a un $21 \%$ cuando se asocia a NF1 $1^{12-14}$.

La resección quirúrgica completa será la base del tratamiento, siendo el abordaje transcervical el más utilizado, ya que permite extendernos en caso de requerir disección cervical. Los TMVNP pueden ser fácilmente disecados de la arteria carótida, yugular interna o nervios adyacentes, al ser circunscritos y menos vascularizados, sin necesidad de reconstruir la carótida interna como es el caso de los tumores vasculares ${ }^{8}$. La radioterapia posoperatoria se debe evaluar caso a caso, ya que se consideran radiorresistentes. Algunos autores recomiendan indicarla tras una recidiva local, sin embargo, no se ha demostrado que aumente la sobrevida global. Por otro lado, el uso de quimioterapia es controvertido, con respuestas favorables solo ocasionalmente. Se ha utilizado con éxito en sarcomas de tejidos blandos fuera del área de cabeza y cuello, por lo que podría ser una opción para evaluar en tumores fuera del alcance quirúrgico y en caso de recidivas ${ }^{5,12,13}$.

\section{Conclusión}

El TMVNP corresponde a un tumor raro, de comportamiento localmente agresivo, las recidivas son frecuentes y pueden dar metástasis a distancia ${ }^{2,6}$. Se debe tener una alta sospecha en caso de antecedentes de NF1 y síntomas sugerentes de déficit del nervio vago. El apoyo imagenológico es crucial para el diagnóstico, así como para planificar el abordaje quirúrgico, el cual dependerá del tamaño tumoral, relación con los grandes vasos y cercanía a la base de cráneo. El tratamiento consiste en la extirpación quirúrgica completa, siendo el predictor más importante de supervivencia. El uso de radio y/o quimioterapia adyuvante se recomienda caso a caso de acuerdo al riesgo de recidiva local, enfermedad residual, tamaño del tumor y grado histológico.

\section{Bibliografía}

1. Arbia Z, Issam S. Malignant peripheral nerve sheath tumor of intracranial nerve: A case series review. Auris Nasus Larynx. 2010;37:539-545. doi: 10.1016/j. anl.2010.02.009.

2. Gaurav G, Allen M. Malignant peripheral nerve sheath tumors. Neurosurg Clin N. 2008;19:533-43. doi: 10.1016/j.nec.2008.07.004.

3. Hajdu SI. Peripheral nerve sheath tumors. Histogenesis, classification, and prognosis. Cancer. 1993;72(12):3549-3552. doi: 10.1002/1097-0142(19931215)72:12<3549::aidcncr2820721202>3.0.co;2-y.

4. Parizel P, Simoens W, Matos C, Verstraete K. Tumors of peripheral nerves. Imaging of Soft Tissue Tumors, 3rd edition. Heidelberg, Germany:Springer, 2006:271-298. doi: 10.1007/978-3-662-07856-3_17.

5. Amir M, Oliver B, Ben H, Wolfgang D, Ulrike B. Malignant peripheral nerve sheath tumors of the head and neck: management of 10 cases and literature review. Head Neck. 2007;29:439-445. doi: 10.1002/ hed.20537.

6. Thway K, Fisher C. Malignant peripheral nerve sheath tumor: pathology and genetics. Ann Diagn Pathol. 2014;18(2):109-116. doi: 10.1016/j. anndiagpath.2013.10.007.

7. Stark A, Buhl R, Hugo H, Mehdorn H. Malignant peripheral nerve sheath tumours report of 8 cases and review of the literature. Acta Neurochir. 2001;143:357364. doi: 10.1007/s007010170090.

8. Lee RM, Ong CP, Jacobsen AS, Chan MY, Hwang WS. Malignant peripheral nerve sheath tumor mimicking carotid body tumor--case report and review. J Pediatr Surg. 2011;46(3):554-558. doi: 10.1016/j.jpedsurg.2010.11.029.

9. Derlin T, Tornquist K, Münster S, et al. Comparative effectiveness of 18F-FDG PET/CT versus wholebody MRI for detection of malignant peripheral nerve sheath tumors in neurofibromatosis type 1. Clin Nucl Med. 2013;38(1):e19-e25. doi: 10.1097/ RLU.0b013e318266ce84.

10. Broski M, Johnson G, Howe B, et al. Evaluation of 18 F-FDG PET and MRI in differentiating benign and 


\section{CASO CLÍNICO}

malignant peripheral nerve sheath tumors. Skeletal Radiol. 2016;45:1097-1105. doi: 10.1007/s00256-0162394-7.

11. Schupp D, Mukherjee D, Sharma G. Schwannoma of the vagus nerve masquerading as a carotid body tumor. Vascular. 2009;17:222-225. doi: 10.2310/6670.2008.00055.

12. Chin-Ta L, Tsai-Wang H, Shin N, Shih-Chun L. Treatment of a malignant peripheral nerve sheath tumor. Onkologie. 2009;32:503-505. doi: 10.1159/000226591.
13. Wanebo JE, Malik JM, VandenBerg SR, Wanebo HJ, Driesen N, Persing JA. Malignant peripheral nerve sheath tumors. A clinicopathologic study of 28 cases. Cancer. 1993;71(4):1247-1253. doi: 10.1002/1097-0142(19930215)71:4<1247::aidcncr2820710413>3.0.co;2-s.

14. Sabesan T, Hussein K, Ilankovan V. Malignant peripheral nerve sheath tumour of the parapharyngeal space in a patient with neurofibromatosis type 1. Br J Oral Maxillofac Surg. 2008;46(7):585-587. doi: 10.1016/j.bjoms.2008.02.003. 